(stages I and II) were diagnosed asymptomatically by a chance visit to a clinic or on ear screening. Others were diagnosed following a complaint of hearing loss, acute otitis media, or otitis media with effusion. The location of congenital cholesteatoma varied somewhat by stage. In stage I congenital cholesteatoma, the most frequent location was behind the anterior-superior quadrant of the tympanic membrane; however, in stage III congenital cholesteatoma, it was behind the posterior-superior quadrant. All patients were treated surgically. Recurrence was detected in 11 of the 87 patients (12.7\%). Recurrent lesions were removed during revision surgery.

Conclusions: Potsic's staging system is a clinically useful procedure for evaluating the extent of congenital cholesteatoma. As the classification is simple, and the stage is easily determined based on otoscopic and CT findings.

doi:10.1017/S0022215116006526

\section{ID: IP156}

The application of endoscopes and microscopes to middle ear surgery

\section{Presenting Author: Hiroshi Ogawa}

Hiroshi Ogawa, Shuji Yokoyama, Tomohiko Ymauchi

Fukushima Medical University Aizu Medical

Center

Learning Objectives: The ratios of microscope and endoscopic use in middle ear surgery in our hospital were investigated, and the effective application of microscopes and endoscopes in middle ear considered.

Objective: The ratios of microscope and endoscopic use in middle ear surgery in our hospital were investigated, and the effective application of microscopes and endoscopes in middle ear considered.

Materials and methods: Middle ear surgery was performed for 63 cases of chronic otitis media with cholesteatoma and 40 cases of chronic otitis media without cholesteatoma in our hospital over a five year period from October, 2011 to September, 2015. The medical records for the 103 patients were reviewed.

Results: Thirty-seven of the 63 cases of chronic otitis media with cholesteatoma underwent surgery with a microscope alone, 15 cases with a combination of microscope and endoscope, and 11 with endoscope alone. Eighteen of the 40 cases of chronic otitis media without cholesteatoma underwent surgery with a microscope alone, 7 cases with a combination of microscope and endoscope, and 15 with endoscope alone. Surgery was performed with an endoscope alone for localized cholesteatoma in the tympanic cavity and for cases in which the edge of the perforation could not be evaluated under microscopic observation. The cases which were required mastoidectomy or in which the edge of perforation was completely visualized underwent surgery with microscope alone.

Discussion: The endoscope has been used as a tool for improving the visual exposure of hidden structures and deep recesses, obtaining a wider angle of view, and achieving minimally invasive intervention. However, its usage during surgery is limited due to its one-hand operation in comparison with surgery under microscopic observation.

Conclusion: The ratios of microscope and endoscope use during middle ear surgery in our hospital were investigated. Improvement in surgical results can be expected by undertaking interventions with an understanding of the advantages and disadvantages of each instrument.

Ethics Committee Approval: Ethics committee approval was received for this study from the local institutional review board $(2015 / 2556)$.

doi:10.1017/S0022215116006538

\section{ID: IP157}

Marked Hearing Improvement After Surgical Removal of Vestibular Schwannoma With Profound Hearing Loss

Presenting Author: Sejoon Oh

Sejoon $\mathrm{Oh}$

Pusan National University Hospital

Learning Objectives: The preoperative hearing status is one of the important factors to determine the method of surgical approach to the vestibular schwannoma. It has been widely recognized that the hearing preservation surgery is not valuable if the patient has no serviceable hearing. The worldwide reported cases of hearing improvement after surgical removal of vestibular schwannoma with profound hearing disturbance are extremely rare so far. The authors have experienced a significant hearing improvement after surgical removal of vestibular schwannoma with preoperative unilateral total deafness but normal otoacoustic emission response, therefore we should consult the OAE response when diciding the approach method of vestibular schwannoma surgery.

doi:10.1017/S002221511600654X

\section{ID: IP158}

Endoscopic Autologous Cartilage

Injection for the Patulous Eustachian tube 
Presenting Author: Sejoon Oh

Sejoon Oh, Soo-Keun Kong

Pusan National University Hospital

\section{Learning Objectives:}

Patulous eustachian tube (PET) can have a significant negative impact on a patient's quality of life. Several methods of surgical management can be an option to treat PET, and our objective is to evaluate the safety and efficacy of autologous cartilage injection in patients with PET. Thirty-three ears of twenty-five patients with chronic PET refractory to conservative treatment were enrolled to this study. Autologous tragal cartilage was harvested, and chopped into fine pieces to allow its injection using a 1cc Bruening syringe. Endoscopic cartilage injection was performed submucosally into the anterior $(0.5$ $\mathrm{mL})$ and posterior aspects $(0.5 \mathrm{~mL})$ of the nasopharyngeal ET under local anesthesia in an operating room. Patients were evaluated postoperatively by nasal endoscopy and by interview to document symptoms. Successful treatment was defined as complete relief or significant improvement plus satisfaction with treatment. The only complication that occurred was temporary otitis media with effusion in one ear. Inferior turbinate reduction was performed in three ears with accompanying nasal septal deviation or turbinate hypertrophy to allow better nasopharyngeal ET visualization. After autologous cartilage injection, the successful treatment rate, as determined by subjective autophony symptoms, was $69.7 \%(23 / 33)$. The average follow-up period was 25.2 months. Autologous cartilage injection is a minimally invasive technique that has been used by the authors to successfully treat patulous eustachian tube. The described procedure was found to provide a good overall success rate without long-term complications.

doi:10.1017/S0022215116006551

\section{ID: IP159}

\section{Surgical results of transcanal endoscopic} ear surgery in chronic otitis media.

Presenting Author: Masafumi Ohki

Masafumi Ohki, Masatoshi Takashima, Yuka Kitano, Atsushi Tahara, Sunao Tanaka, Tsutomu Nomura, Atsushi Ohata, Shigeru Kikuchi

Saitama Medical Center

Learning Objectives: To endoscopically examine surgical results for chronic otitis media after endoscopical ear surgery comparing with mocroscopic ear surgery

Introduction: Middle ear surgery has commonly been treated using a surgical microscope. A binocular stereomicroscope has often been used in ear surgery because this instrument offers many advantages including binocular stereoscopic vision of the surgical field; no obstruction of the view by blood, mucus, or bone dust on the lens; high magnification, besides being hands-free. Conversely, the narrow-angle view is a disadvantage of using a microscope for middle ear surgery. Moreover, there are several blind areas behind important structures, such as the facial nerve, that cannot be avoided. The use of an endoscope can offer several advantages over the use of a microscope during middle ear surgery, particularly the wider field of view. Therefore, endoscopic ear surgery, especially transcanal approach, has been developing recently. The aim of this study is to endoscopically examine surgical results for chronic otitis media after endoscopical ear surgery comparing with mocroscopic ear surgery.

Methods: Sixty-nine consecutive patients who underwent tympanoplasty for chronic otitis media. Transcanal endoscopic ear surgery was performed in 25 patients, and postauricular incision microscopic ear surgery in 44. Hearing outcome of air conduction threshold, bone conduction threshold, air-bone gap was assessed.

Results: Surgical results of hearing levels after transcanal endoscopic ear surgery was significantly better than postauricular incision microscopic ear surgery. Transcanal endoscopic ear surgery is advantageous to approach to the attic and perform tympanoplasty for sound transmission.

Conclusions: Surgical results of transcanal endoscopic tympanoplasty for chronic otitis media were excellent.

\section{doi:10.1017/S0022215116006563}

\section{ID: IP160}

\section{Postoperative residual cases in pediatric acquired cholesteatoma}

\section{Presenting Author: Shinsuke Ohshima}

Shinsuke Ohshima ${ }^{1}$, Yuka Morita ${ }^{1}$, Kuniyuki Takahashi ${ }^{1}$, Shuji Izumi ${ }^{1}$, Yamato Kubota ${ }^{1}$, Yutaka Yamamoto ${ }^{2}$, Sugata Takahashi ${ }^{1}$, Arata Horii ${ }^{1}$

${ }^{1}$ Niigata University Graduate School of Medical and Dental Sciences, ${ }^{2}$ The Jikei

University School of Medicine

\section{Learning Objectives:}

Introduction: Acquired cholesteatoma is more aggressive in children than in adults. Despite the aggressive behaviour, radical treatment such as canal wall down technique was less performed to reduce cavity problem which requires endless care. This results in high rate of residues and recurrence. We focused in this study on reducing residues in pediatric acquired cholesteatoma surgery and explored risk factors of residual lesions.

Methods: Medical charts of 39 children under 15 years old with acquired cholesteatoma were retrospectively reviewed. Various factors were compared between the residual cholesteatoma $(+)$ and (-) groups: surgical procedures, type of cholesteatoma, number of primary sites of cholesteatoma at surgery ( $\mathrm{P}$, protympanum; $\mathrm{T}$, tympanic cavity; $\mathrm{A}$, attic; $\mathrm{M}$, mastoid), development of mastoid air cells, and the status of stapes. Residue $(+)$ was defined if residual lesion was found after one-stage surgery or planned two-stage surgery, but not during second-look operation. 